Received 00th January 20xx, Accepted 00th January 20xx DOI: $10.1039 / \times 0 \times x 00000 x$

\title{
Matching sensitivity to abundance: High resolution immuno-mass spectrometry imaging of lanthanide labels and endogenous elements in the murine brain
}

\author{
David Clases*a, Raquel Gonzalez de Vega ${ }^{a}$, Sabrina Funke ${ }^{b}$, Thomas E. Lockwooda, Mika T. \\ Westerhausen ${ }^{a}$, R. Verena Taudtec, Paul A. Adlard ${ }^{d}$, Philip A. Doble*a
}

\begin{abstract}
This work introduces a new method for immuno-mass spectrometry imaging via quadrupole-based laser ablation inductively coupled plasma - mass spectromery instruments that is matched to the abundance of elements in biological tissues. Manipulation of ion-optics and quadrupole mass filter parameters provided increased transmission of low level highmass elements, which are typically used as labels for antibodies, at the expense of highly abundant endogenous low-mass elements. Transmission of mid-mass elements such as the transition metals was only slightly affected. The implications for mass resolution and background signals are critically discussed and signal to noise ratios and imaging capabilities are compared to those of obtained from a standard method. This novel approach resulted in a 6-fold improved signal to noise ratio for lanthanides that are routinely used as elemental labels for antibodies to target protein distributions in biological tissues. This increase in signal intensity, enhanced contrasts, lower limits of detection and the capability to improve spatial resolution. All together, this contributed to enhanced imaging capabilities and trace analyses, as demonstrated by imaging murine brain sections of the hippocampal system and substantia nigra.
\end{abstract}

\section{Introduction}

The detection and quantification of endogenous elements and proteins in biological tissues is essential to interrogate biological processes and provide useful information for numerous research endeavours such as neuroscience ${ }^{1}$, diagnostic surgical pathology 2,3 , drug discovery workflows ${ }^{4-6}$ and infection control?. Initial efforts to visualise spatial distributions of proteins in biological tissues date back to the 1940s when Coons et al. developed methods for the conjugation of antibodies and fluorescent labels which is now known as immunohistochemistry $(\mathrm{IHC})^{8}$. Although IHC-based methods have improved dramatically over the past decades ${ }^{9-11}$, multiplexed visualisation of low abundant proteins is limited due to problems such as photo-bleaching ${ }^{12}$, co-localisation of proteins, and matrix or background autofluorescence ${ }^{13,14}$. Emerging novel methods that combine IHC and mass spectrometry have considerable potential to address the

\footnotetext{
a. The Atomic Medicine Initiative, University of Technology Sydney, Sydney, NSW, Australia

b. Institute of Inorganic and Analytical Chemistry, University of Münster, Münster, Germany

c. Institute for Experimental and Clinical Pharmacology and Toxicology, Friedrich-

Alexander-University, Erlangen - Nürnberg, Germany

d. The Florey Institute of Neuroscience and Mental Health, The Melbourne Dementia

Research Centre, The University of Melbourne, Parkville, Victoria, Australia
}

\section{* Corresponding authors:}

David Clases e-mail: David.Clases@uts.edu.au

Philip Doble, e-mail: Philip.Doble@uts.edu.au spatiotemporal and quantitative challenges presented by the measurement of proteins in biological systems ${ }^{15-19}$. IHC was first integrated into the workflow of mass imaging via laser ablation - inductively coupled plasma - mass spectrometry (LAICP-MS) by Zhang et al. for the investigation of thyroid stimulating hormone ${ }^{20}$. Since then, various labelling protocols that tag one or several metals to antibodies and different protocols to characterise and validate labelling processes have been developed ${ }^{14,19,21}$. Up to 32 different proteins may be labelled with different metal tags and detected with high sensitivity and subcellular lateral resolution $(1 \mu \mathrm{m})^{22}$. Commercially available kits provide straight forward labelling and staining procedures ${ }^{21}$, and different standardisation approaches contribute to robust quantification and the mitigation of instrumental signal drifts ${ }^{23,24}$.

Spatial resolution is limited by the sensitivity of the ICP-MS method which is proportional to the square of the laser beam spot radius, i.e. reducing the spot size by half results in four times less material per ablation event ${ }^{25,26}$. This is a limitation for the interrogation of brain tissue where many anatomical and cellular features require high resolution imaging of proteins and/or essential transition elements at trace concentrations. For example, co-localisation of iron ( $\mathrm{Fe}$ ) and dopamine can be measured in the substantia nigra pars compacta to predict the risk of parkinsonian neurodegeneration ${ }^{27}$. This co-localisation of Fe and dopamine promotes the generation of reactive oxygen and quinone species causing the destruction of dopaminergic cells ${ }^{27-31}$. Whilst Fe among other trace metals may be targeted by conventional (quantitative) LA-ICP-MS ${ }^{32}$, dopamine may be measured indirectly by targeting tyrosine hydroxylase (TH) with 
immuno-mass spectrometry imaging (iMSI). TH is involved in the production of dopamine and catalyses rate-limiting reactions for dopamine synthesis. Furthermore, there are numerous anatomical features that require high-resolution imaging. One such example is the hippocampus in murine brains which has features with dimensions below $50 \mu \mathrm{m}$ that would require several data points for adequate structural clarity. Therefore, iMSI requires improvements to increase sensitivity, lower limits of detection (LODs) and increase signal to noise ratios (SNRs) to resolve low abundant proteins and trace elements in such structures.

In routine analysis, method parameters for LA-ICP-MS are ideally set to values for uniform transmission of all masses. However, ions with different masses have different responses to changes in the ion optics voltages which are typically optimised for the mid-mass range at the expense of the transmission of heavier and lighter elements ${ }^{33}$. While this is a suitable compromise for most applications, standard tunes are sub-optimal when endogenous metals and low abundant proteins are targeted by iMSI. In many cases, analyte concentrations may be estimated prior to analysis and specialised methods optimised for transmission of low abundant analytes at the expense of those expected to be highly abundant and well above the LOD can improve imaging quality and LODs. The substantia nigra and the hippocampus are two such examples, known to be rich in phosphorus and to contain Fe well above LODs (higher than $\left.20 \mu \mathrm{g} \mathrm{g}^{-1}\right)^{27}$. However, the protein content is often variable at levels within or below the $\mathrm{pg} \mathrm{g}^{-1}$ range. As a rule of thumb, biological samples contain a high abundance of low mass elements such as sulphur (S), phosphorus $(\mathrm{P})$ and sodium $(\mathrm{Na})$, whereas essential mid mass transition metals such as $\mathrm{Fe}$, copper $(\mathrm{Cu})$ and zinc $(\mathrm{Zn})$ are present at much lower concentrations. The concentration of lanthanides typically coupled to antibodies for iMSI are dependent on the abundance of the targeted protein and are therefore highly variable. Potential issues associated with these variabilities of concentration may be overcome by manipulation of the sensitivity for mid and high-mass elements, at the expense of low mass elements as shown in Figure 1.

This paper describes the development of a high transmission method for quadrupole-based LA-ICP-MS suitable for the spatial determination of metals introduced into biological tissues via antibody probes, as well as endogenous elements.

\section{Experimental}

\section{Chemicals and consumables}

Anti-tyrosine hydroxylase was purchased from In vitro Technologies (Victoria, Australia) and a ${ }^{172} \mathrm{Yb}$ MAXPAR ${ }^{\mathrm{TM}}$ labelling kit from Millennium Science (Victoria, Australia). Centrifugal filters with mass cut offs at $3 \mathrm{kDa}$ and $50 \mathrm{kDa}$ were obtained from Sigma Aldrich (Amicon ${ }^{\circledR}$ Ultra-0.5, Darmstadt, Germany).

\section{Standard Preparation}

The method was developed and optimised for the calculation of SNRs using homogenous laboratory-made matrix-matched standards manufactured according to the protocol by Westerhausen et al. ${ }^{24}$. Briefly, $100 \mathrm{mg}$ high purity gelatin from bovine skin (Sigma Aldrich) was mixed with $900 \mu \mathrm{L}$ of ${ }^{172} \mathrm{Yb}, \mathrm{Mn}$, $\mathrm{Cu}, \mathrm{Na}$ and $\mathrm{P}$ diluted in $100 \mathrm{mM}$ Tris- $\mathrm{HCl}$ buffer (pH 7.4), $10 \mathrm{mM}$ EDTA and $1 \% \mathrm{w} / \mathrm{w}$ polyethylene glycol $(\mathrm{Mn} 400)$ prepared in Milli-Q water (18.2 M $\Omega \mathrm{cm}-1$ at $25^{\circ} \mathrm{C}$, Arium Pro Vf, Sartorius, Goettingen, Germany).

The spiked gelatin was heated to $55^{\circ} \mathrm{C}$ and filled into wells (HybriWell ${ }^{\mathrm{TM}}, 50 \mu \mathrm{L}, 9.8 \mathrm{~mm} \times 20 \mathrm{~mm} \times 0.25 \mathrm{~mm}$ ) which produced a thin gelatin film with a defined thickness $(25 \mu \mathrm{m})$, homogeneous metal distribution and a smooth topography ${ }^{24}$.

\section{Sample Preparation}

Wild type C57BI/6 murine tissue was provided from the Florey Institute of Neuroscience and Mental Health (University of Melbourne, Australia). Animal experiments were approved by the Florey Institute Animal Ethics committee and were conducted in accordance with the Australian Code of Practice for the Care and Use of Animals for Scientific Purposes as described by the National Health and Medical Research Council of Australia. All animals had free access to food and water and were group-housed in individually ventilated cages in The Florey Institute Animal Facility under controlled temperature $\left(22 \pm 2^{\circ} \mathrm{C}\right)$ and lighting (14:10 hours light/dark cycle) conditions. Animals were euthanized with sodium pentobarbitone $(80 \mathrm{mg} / \mathrm{kg})$ via intraperitoneal injection, followed by transcardial perfusion with $0.1 \mathrm{M}$ phosphate buffered saline (PBS). The brains were then immediately frozen to conserve the elemental distribution and content and sectioned $(30 \mu \mathrm{m})$ on a cryotome (CryoStar ${ }^{\mathrm{TM}}$, Thermo Fisher Scientific, North Ryde, NSW, Australia) and mounted on microscope glass slides (Superfrost ${ }^{\mathrm{T} M}$, Thermo Fisher Scientific, North Ryde, NSW, Australia). Brain sections were stored at $-80^{\circ} \mathrm{C}$.

The antibody was labelled by reduction with a $0.4 \mathrm{mM}$ tris(2carboxyethyl)phosphine hydrochloride solution and subsequent conjugation of metal loaded polymers to the $F_{c}$ region, according to the standard Fluidigm protocol (PRD 002, Version 10). The labelled antibody was diluted $1: 2$ in stabiliser solution and diluted again 1:1000 for staining purposes.

The mouse brain tissue was fixed with $2 \%$ paraformaldehyde for a duration of 5 minutes. After three washing steps, a blocking solution comprising of $1 \%(\mathrm{w} / \mathrm{v})$ bovine serum albumin (BSA; Sigma Aldrich, Sydney, Australia), 0.3\% Triton X-100, 3\% (v/v) normal goat serum (Sigma Aldrich) in phosphate-buffered saline was applied for 30 minutes on the tissue. After an additional washing step, the labelled antibody solution was incubated overnight before washing and air drying the specimen. Instrumentation and Experimental Parameters 


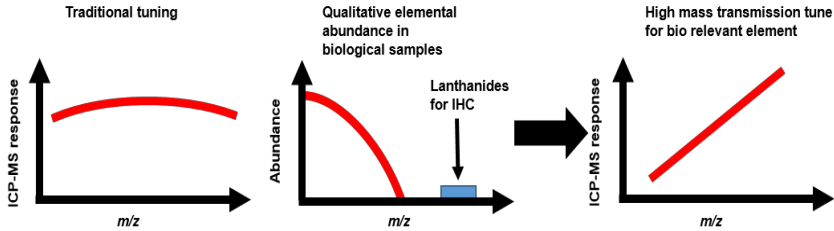

Figure 1. (A) Conventional tuning with similar transmission/sensitivity of all masses. (B) Simplified illustration of the abundance of elements in a typica biological soft tissue matrix. (C) An idealised tune method with a transmission endogenous (mid-mass) elements.

Method development was performed on a 7500-series cS quadrupole ICP-MS (Agilent Technologies, Santa Clara, USA) instrument equipped with nickel sampler and skimmer cones and operated using ChemStation software (Agilent Technologies). The operation of a dynamic reaction cell was not necessary due to reduced polyatomic interferences under dryplasma conditions in LA-ICP-MS. An LSX-213 G2+ laser system from Teledyne CETAC Technologies (Omaha, NE, USA) was equipped with an "aerosol rapid introduction system" (ARIS, Teledyne CETAC Technologies, USA) for improving wash-out times and improving lateral resolution and was operated at $20 \mathrm{~Hz}$. Ultra-pure helium (BOC, North Ryde, NSW, Australia) was used as carrier gas with a flow rate of $0.4 \mathrm{~L} / \mathrm{min}(0.25 \mathrm{~L} / \mathrm{min}$ cell gas, $0.15 \mathrm{~L} / \mathrm{min}$ cup gas).

To achieve higher sensitivity for high resolution imaging of neurological structures, a 7700-series quadrupole ICP-MS (Agilent Technologies) instrument was equipped with CS lenses, nickel sampler and skimmer cones, operated using MassHunter software (Agilent Technologies) and coupled to a NWR193 Laser Ablation System operated at $40 \mathrm{~Hz}$ (Kenelec Scientific, Frenchs Forest, Australia). Argon was used as carrier gas with $1.10 \mathrm{~L} / \mathrm{min}$.

The laser beam spot sizes and scan speeds were adjusted to a ratio of 1:4 and the quadrupole's duty cycle was set to $250 \mathrm{~ms}$ to produce squared pixels. The laser beam spot size was set to $50 \mu \mathrm{m}$ for initial method development and was decreased to $20 \mu \mathrm{m}$ to image the limbic system and substantia nigra, and $5 \mu \mathrm{m}$ for high resolution imaging of the hippocampus. The laser fluence was optimised to $2.04 \mathrm{~J} / \mathrm{cm}^{2}$.

\section{Initial tuning parameters}

The ICP-MS hardware was initially tuned to the highest signal to noise ratios (SNRs) within intervals recommended by the manufacturer (Agilent 7500 ICP-MS ChemStation Operator's Manual, Tuning). The quadrupole's resolution was set to provide a uniform mass resolution of 0.70 atomic mass units (amu) at $10 \%$ mass peak height over the entire mass range. The parameters of this standard tune are listed in Table 1, together with the manufacturer's recommended adjustment intervals and the modified values of the high transmission method. The SNRs were calculated using the background intensities of the laser's shutter blank and the signal intensity from a matrix matched standard or sample, respectively.
Table 1. Overview of adjustable tune parameters of the 7500cs series ICP-MS. A standard tune was generated to provide uniform sensitivity over the entire mass range. The parameters for the standard tune were adjusted within the recommended values. To generate a high transmission tune, all parameters were adjusted to provide high transmission/sensitivity for high mass elements, with some values outside the recommend range.

\begin{tabular}{|c|c|c|c|}
\hline Parameter & $\begin{array}{l}\text { Standard } \\
\text { method }\end{array}$ & $\begin{array}{c}\text { Recommended } \\
\text { adjustment } \\
\text { range }\end{array}$ & $\begin{array}{c}\text { High } \\
\text { transmission } \\
\text { method }\end{array}$ \\
\hline Extract 1 & $5.4 \mathrm{~V}$ & 4 to $8 \mathrm{~V}$ & $5.4 \mathrm{~V}$ \\
\hline Extract 2 & $-100 \mathrm{~V}$ & -150 to $-60 \mathrm{~V}$ & $-120 \mathrm{~V}$ \\
\hline Omega Bias-cs & $-32 \mathrm{~V}$ & -45 to $-25 \mathrm{~V}$ & $-50 \mathrm{~V}$ \\
\hline Omega Lens-cs & $8.6 \mathrm{~V}$ & 5 to $10 \mathrm{~V}$ & $7.6 \mathrm{~V}$ \\
\hline Cell Entrance & $-26 \mathrm{~V}$ & -40 to $-20 \mathrm{~V}$ & $-46 \mathrm{~V}$ \\
\hline QP Focus & $2 \mathrm{~V}$ & -2 to $5 \mathrm{~V}$ & $4 \mathrm{~V}$ \\
\hline Cell Exit & $-30 \mathrm{~V}$ & -45 to $-20 \mathrm{~V}$ & $-40 \mathrm{~V}$ \\
\hline OctpRF & $150 \mathrm{~V}$ & 100 to $200 \mathrm{~V}$ & $160 \mathrm{~V}$ \\
\hline OctpBias & $-6 V$ & -12 to $-6 \mathrm{~V}$ & $-11.2 \mathrm{~V}$ \\
\hline QP Bias & $-3 V$ & -5 to $-3 \mathrm{~V}$ & $-20 \mathrm{~V}$ \\
\hline $\begin{array}{l}\text { Mass resolution } \\
\text { (width at } 10 \% \text { ) }\end{array}$ & $\begin{array}{c}\text { m/z 172: } \\
0.7 \text { amu, } \\
\text { m/z 55: } \\
0.7 \mathrm{amu}, \\
\mathrm{m} / \mathrm{z} 23: \\
0.7 \mathrm{amu}\end{array}$ & $\begin{array}{c}0.65 \text { to } 0.8 \\
\text { amu }\end{array}$ & $\begin{array}{c}\mathrm{m} / \mathrm{z} 172: \\
3.69 \mathrm{amu}, \\
\mathrm{m} / \mathrm{z} 55: \\
0.64 \mathrm{amu}, \\
\mathrm{m} / \mathrm{z} 23: \\
0.34 \mathrm{amu}\end{array}$ \\
\hline
\end{tabular}

\section{Image generation}

Images were processed by ImaJar 3.49 developed by Robin Schmid (University of Muenster, Germany) and pew ${ }^{2}$ 0.7.0, an in-house developed software (https://github.com/djdt/pewpew). All images were processed by applying a bicubic interpolation algorithm. Noise was calculated by averaging the intensities obtained during the laser warm up (closed shutter). SNRs were calculated by dividing all signal intensities by the average noise value.

\section{Results and Discussion}

\section{Method development and basic considerations}

Imaging murine brain anatomical structures are challenging as high sensitivity is required in combination with high resolution - two parameters that correlate inversely. Here, we present a
A

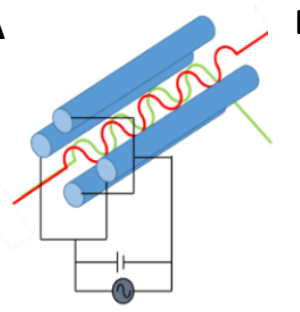

B

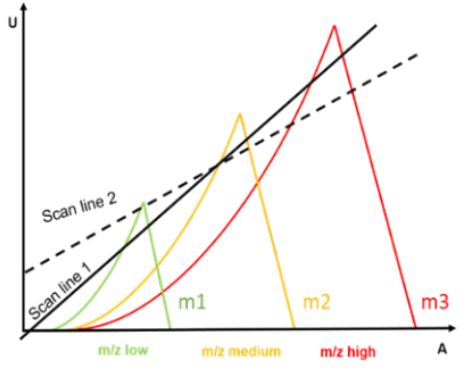

Figure 2. Operation principals of a quadrupole. The combination of a direct current voltage $(U)$ and a radio frequency voltage (A) determines the transmission bandwidt of selected $\mathrm{m} / \mathrm{z}$. Changing the scanning the transmission for high mass elements 
new method that tailors the sensitivity and SNRs to the elemental abundance in brain tissue by specialised optimisation of the ion optics and mass analyser. The transmission, background and selectivity of ICP-MS depend on a variety of synergistic parameters, where the manipulation of an individual parameter can change the optimum working range of others.

A typical quadrupole mass analyser consists of 4 hyperbolic rods (Fig. 2 (A)) designed to sequentially filter ionised masses and is operated with a specific set of direct current voltages $(U)$ and RF-voltages (A) for the analysis of different mass to charge ratio $(\mathrm{m} / \mathrm{z})$, respectively. Each $\mathrm{m} / \mathrm{z}$ has a unique set of $U$ and $A$ combinations that generate a stable trajectory through the quadrupole. Consider Fig. 2 (B), which shows regions of transmission stability of ions at low, mid, and high masses, represented by the green, yellow and red zones, where $\mathrm{m} 1<$ $\mathrm{m} 2<\mathrm{m} 3$. All $\mathrm{U}$ and $\mathrm{A}$ combinations that a quadrupole typically operates are aligned on one straight scan line. The slope and intercept of the scan line determine the mass resolution and ion transmission of the quadrupole, whereas mass resolution and transmission correlate inversely. The scan line conventionally intersects the tips of the stability areas, whereas the area of the truncated tips correlate positively with transmission and negatively with mass resolution ${ }^{34}$. To achieve uniform mass resolution and transmission over the entire mass range, the scan line is typically set as shown for scan line 1 in Fig.2 (B). Changing slope and intercept of the scan line (scan line 2 in Fig. 2 (B)) will change the bandwidths of the quadrupole and affect transmission and mass resolution and the sensitivity and background signal ${ }^{35}$. Increasing the intercept and reducing the slope of the scan line reduces the transmission of low $\mathrm{m} / \mathrm{z}$ and increase it for high $\mathrm{m} / \mathrm{z}$, while mid $\mathrm{m} / \mathrm{z}$ are less affected.

Traditionally, a quadrupole is tuned to resolve each mass peak with a signal width of $0.65-0.8$ amu at $10 \%$ of peak height to ensure that signals from adjacent masses do not spectrally overlap. However, when modifying scan lines, such as in scan line 2, mass resolution and selectivity is lost in the high mass range, and potential interferences need to be considered for routine analyses. High mass elements are not naturally present in biological specimens, and therefore potential interferences can be essentially ignored. Furthermore, high mass polyatomic interferences in dry plasmas such as those in LA-ICP-MS are unlikely. These factors mean the mass resolution of high mass elements in iMSI is less important and standard methods can be

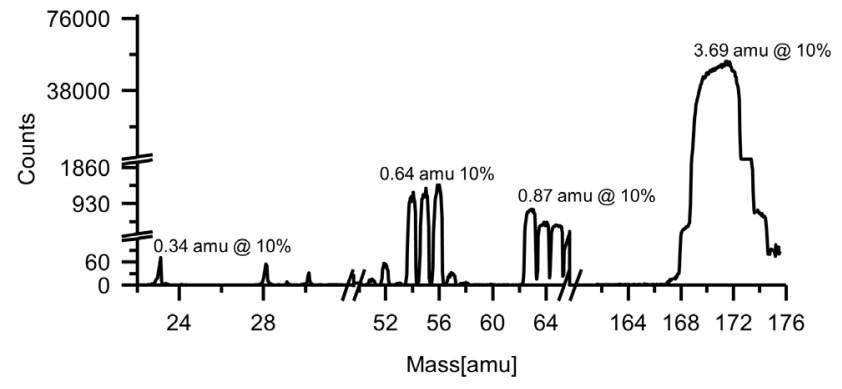

Figure 3. Mass spectrum obtained by LA-ICP-MS of a gelatin standard demonstrating the effect of modifying the parameters of the ion optics and scanning characteristics of the quadrupole. The transmission of low mass elements was decreased while the resolution was increased. Essential mid mass elements were slightly affected. The mass resolution of high masses decreased whilst transmission was increased dramatically.

altered to allow greater transmission of lanthanides. This greatly improves the sensitivity of lanthanide antibody tags, whilst allowing sufficient mass resolution and sensitivity for measurement of endogenous elements.

The high transmission tune for improving the simultaneous measurement of labelled proteins and endogenous elements was developed by ablating solid gelatin standards spiked with $2.5 \mu \mathrm{g} / \mathrm{g}$ of $94 \%$ isotopically enriched ${ }^{172} \mathrm{Yb}$ and between $1-20$ $\mu \mathrm{g} / \mathrm{g}$ of ${ }^{23} \mathrm{Na},{ }^{31} \mathrm{P},{ }^{55} \mathrm{Mn},{ }^{57} \mathrm{Fe},{ }^{63} \mathrm{Cu}$. The ion optics and quadrupole parameters were optimised heuristically and iteratively ( 3 times). The resulting transmission and mass resolution of the target elements are shown in Figure 3. It was observed that the optimal voltage parameters for the ion lenses changed significantly with the bandwidth of the quadrupole. The mass spectrum had an increased mass resolution for ${ }^{23} \mathrm{Na}$ with a peak width (at $10 \%$ of peak's height) of $0.34 \mathrm{amu}$. The mass resolution of ${ }^{55} \mathrm{Mn},{ }^{57} \mathrm{Fe}$ and ${ }^{63} \mathrm{Cu}$ ranged from 0.64 to $0.87 \mathrm{amu}$, whilst the signal corresponding to ${ }^{172} \mathrm{Yb}$ was significantly wider with a peak width of $3.69 \mathrm{amu}$. The asymmetric shape of $\mathrm{Yb}$ resulted from other minor $\mathrm{Yb}$ isotopes which could not be resolved individually under this quadrupole conditions contributing to a combined signal additionally increasing sensitivity. Increases in SNRs can be expected to be even higher when working with non-enriched isotopic tags such as nanoparticles or when targeting naturally abundant lanthanides ${ }^{36}$.

Table 2. Comparison of the relative improvements in signal intensity and background for the standard and high transmission method.

\begin{tabular}{|c|c|c|c|c|c|c|c|}
\hline \multirow[b]{2}{*}{$\mathrm{m} / \mathbf{z}$} & \multicolumn{3}{|c|}{ Standard method } & \multicolumn{3}{|c|}{ High transmission method } & \multirow{2}{*}{$\begin{array}{l}\text { Relative } \\
\text { change of } \\
\text { SNR }\end{array}$} \\
\hline & $\begin{array}{l}\text { Background } \\
\text { [cps] }\end{array}$ & $\begin{array}{c}\text { Signal } \\
\text { [cps] }\end{array}$ & SNR & $\begin{array}{c}\text { Background } \\
\text { [cps] }\end{array}$ & $\begin{array}{c}\text { Signal } \\
\text { [cps] }\end{array}$ & SNR & \\
\hline${ }^{23} \mathrm{Na}$ & 122000 & 1470000 & 12.0 & 43.9 & 317 & 7.23 & 0.60 \\
\hline${ }^{31} \mathbf{p}$ & 680 & 1370 & 2.00 & 2.17 & 3.48 & 1.6 & 0.80 \\
\hline${ }^{55} \mathrm{Mn}$ & 1950 & 32200 & 16.4 & 490 & 10300 & 21.1 & 1.28 \\
\hline${ }^{57} \mathrm{Fe}$ & 610 & 750 & 1.23 & 198 & 307 & 1.55 & 1.26 \\
\hline${ }^{63} \mathrm{Cu}$ & 460 & 20600 & 44.6 & 213 & 6090 & 28.6 & 0.64 \\
\hline${ }^{172} \mathrm{Yb}$ & 1.74 & 217000 & 125000 & 0.976 & 835000 & 855000 & 6.86 \\
\hline
\end{tabular}


The optimised parameters of the high transmission tune are shown in Table 1, and the background signal, response signal and improvements in SNR are shown in Table 2.

The tuning of the ion optics and mass analyser had various effects on different elements. Increasing the transmission and sensitivity for transition metals did not always contribute to increases of SNR and sometimes had the opposite effect. The outcome was dependant on the background intensity of an individual $\mathrm{m} / \mathrm{z}$, which originated from random noise or spectral interferences. The mid masses were most sensitive to undesirable increases in background signal due to the high probability of the formation of argon-based polyatomic interferences, especially Fe. As the implications of changing the bandwidth of the quadrupole were difficult to predict and were different for individual isotopes, the effect on each element of interest was investigated empirically.

The final optimised method reduced the SNR for ${ }^{23} \mathrm{Na},{ }^{31} \mathrm{P}$, and ${ }^{63} \mathrm{Cu}$ by a relative factor of $0.60,0.80$ and 0.64 , respectively. The SNR for ${ }^{55} \mathrm{Mn},{ }^{57} \mathrm{Fe}$ and ${ }^{172} \mathrm{Yb}$ on the other hand were improved by a relative factor of $1.28,1.26$ and 6.86 , respectively. As hypothesised, the adjustment of the scan line around a fixed point located in the mid mass range (represented by line scan 2 in Figure 2(b)) resulted in relatively small changes for mid mass elements but had a large impact on mass resolution and transmission of high masses. The decreased mass resolution led to a single peak for all isotopes of $\mathrm{Yb}$ and was not a disadvantage for this application but must be considered when unit mass resolution is required as for multiplexed analysis. This disadvantage may be mitigated by choosing labels with at least 6-8 amu difference.

\section{Imaging murine brain sections}

The effect of the high transmission method on the analysis of endogenous elements and metals introduced into biological specimens by IHC was demonstrated on murine brain sections in the region of the substantia nigra and the hippocampus. Both areas contain $\mathrm{TH}$ that is responsible for the production of dopamine and may be used as a proxy for the measurement of active dopaminergic cells and is useful for investigations of the pathology of Parkinsonian diseases. TH was labelled with ${ }^{172} \mathrm{Yb}$ and incubated on two consecutive brain sections prior to analysis by the standard method and high transmission method, respectively. Figure 4 compares the result of representative low, mid and high $\mathrm{m} / \mathrm{z}$ values. Values below the limits of detection $(\mathrm{SNR}<3)$ are shown on a grey scale and those above $(S N R \geq 3)$ on a rainbow scale. For the analysis of ${ }^{172} \mathrm{Yb}$, the high transmission method yielded a significant increase in SNR.

To allow objective visual comparisons of SNRs for the analysis of ${ }^{172} \mathrm{Yb}$, the scales' maxima were fixed at a value that contained $99 \%$ of the pixels for both images. The high transmission tune resulted in an approximately 6-fold increased SNR compared to the standard method which was consistent with the improvements shown in Table 2. Fe was investigated as an endogenous mid mass element. ${ }^{57} \mathrm{Fe}$ was not sufficiently abundant to create anatomical contrasts as it was a minor isotope (2.2\%), and ${ }^{56} \mathrm{Fe}$ (92\% isotopic abundance) was targeted

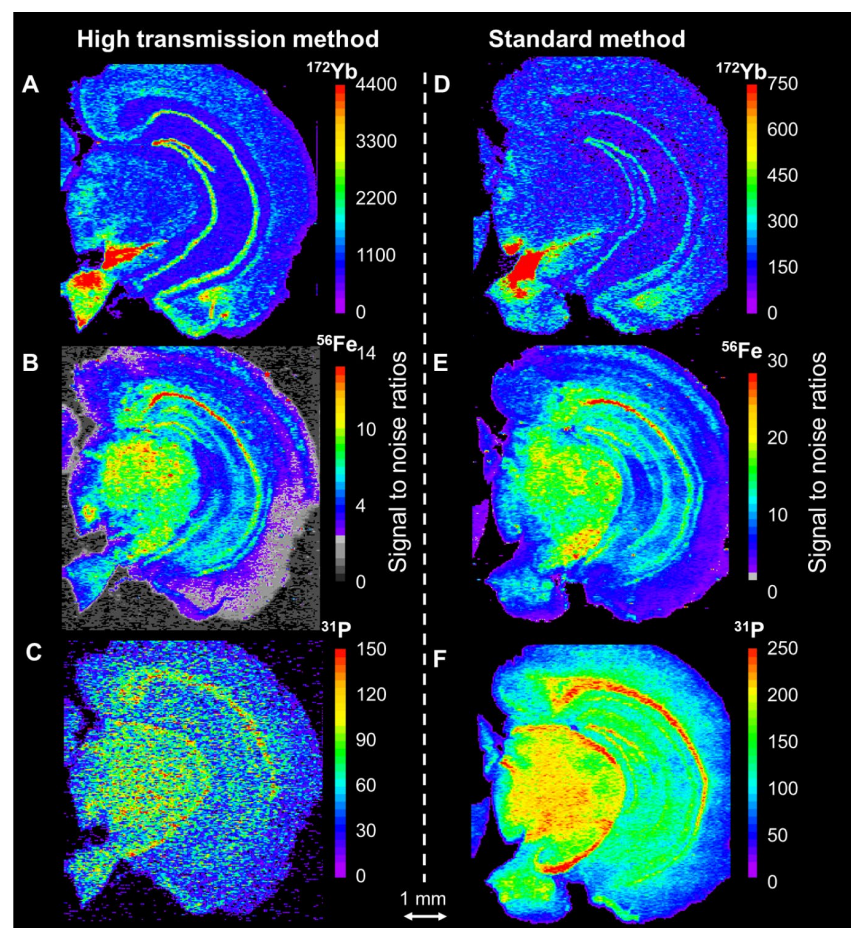

Figure 4. The high transmission method was used to determine the elementa distribution (left column, B: ${ }^{56} \mathrm{Fe}, \mathrm{C}^{31} \mathrm{P}$ ) and TH expression (A) in a IHC stained mouse brain section and was compared with a standard method (right column D: TH, E: ${ }^{56} \mathrm{Fe}$, F: ${ }^{31} \mathrm{P}$,). The high transmission method resulted in up to
approximately 6-fold enhanced SNR for ${ }^{172} \mathrm{Yb}$.

instead. The operation of a dry plasma reduced the abundance of polyatomic interferences negating the need for a collision/reaction cell, as well as providing the optimum conditions for the determination of the targeted lanthanide. Both tunes produced a ${ }^{56} \mathrm{Fe}$ distribution with comparable anatomical structures and hotspots. The SNRs deviated by approximately a factor of 2 between the standard and high transmission method, respectively. For ${ }^{31} \mathrm{P}$, the standard method resulted in SNRs exceeding 250 and resolved several anatomical features within the mouse brain, whereas the high transmission method resulted in inferior SNR and sensitivity due to reduced signal intensity. In conclusion, the high transmission tune improved the SNR for the lanthanide tag, whilst still resolving the distributions of essential highly abundant mid and low mass elements in biological tissue.

\section{High resolution imaging targeting $\mathrm{TH}$ and Fe in the hippocampus and substantia nigra}

The increased transmission improved the overall figures of merit for the analysis of the high-mass elements. This resulted in both lower limits of detection and the possibility to increase spatial resolution, which is of major relevance for low abundant elements and proteins, and visualisation of important microstructures in brain tissues. One such example is the hippocampus, which is critically involved in learning and memory and other higher brain functions. This structure is highly defined with dimensions of 10 to 20 micrometres in some areas in murine tissue. TH in dopaminergic cells in the hippocampus is an important enzyme and valuable biomarker 


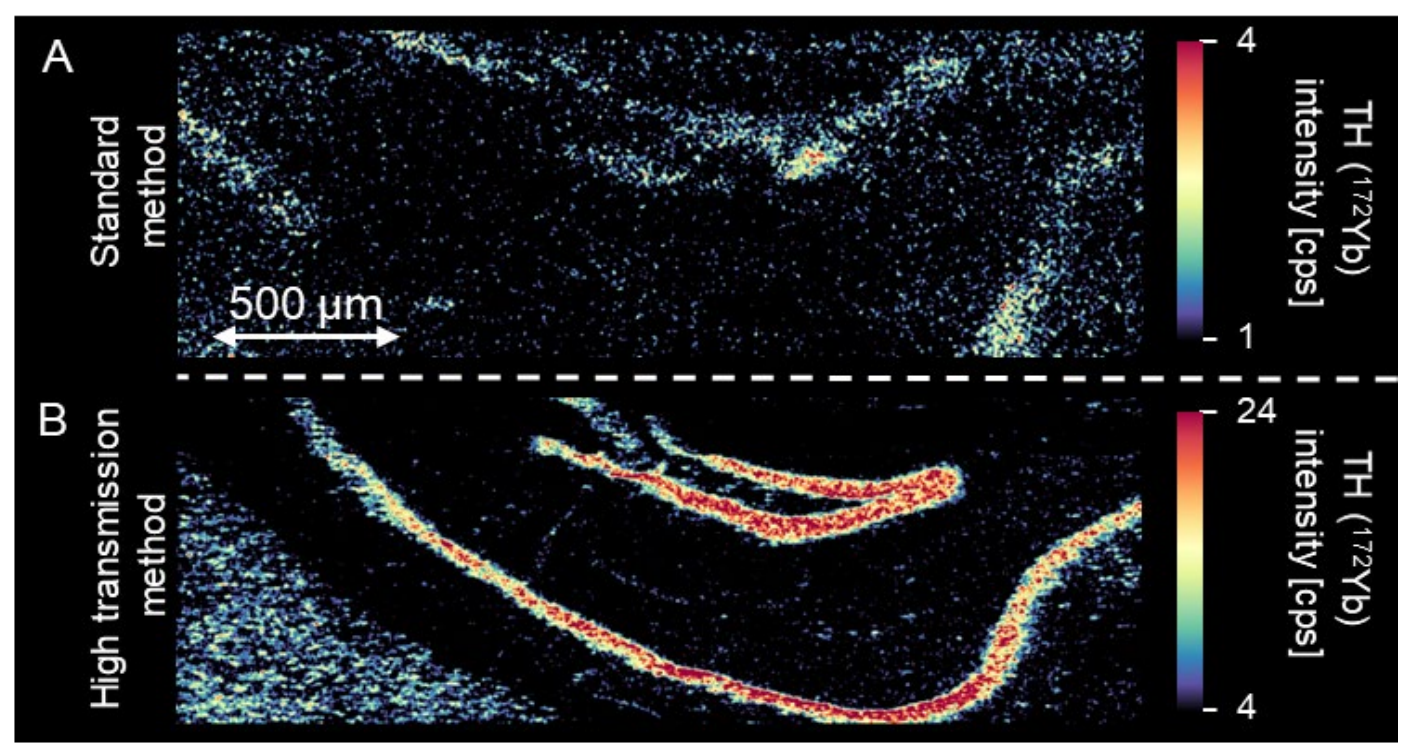

Figure 5. The hippocampal structure was targeted in two consecutive sections of murine brain employing (A); standard method and (B); the high transmission method.

of neurological decline ${ }^{37}$. Two consecutive sections of murine hippocampus were visualised by targeting TH with a laser beam spot size of $5 \mu \mathrm{m}$ producing pixels of $25 \mu \mathrm{m}^{2}$ as shown in Fig. 5 . Here, ${ }^{172} \mathrm{Yb}$ was labelled to polyclonal anti-TH and imaged with the standard method (Fig. 5A), and the high transmission method (Fig. 5B). The latter tune showed vastly improved contrast and SNRs of the cornu ammonis (CA) subfields and dentate gyrus in the hippocampus. Although the high transmission method favoured high masses at the expense of high abundant low mass elements, ion transmission and massresolution of mid-mass elements is almost unchanged. This offers advantages for the combined detection and imaging of endogenous metals and proteins. Typically, biologically relevant

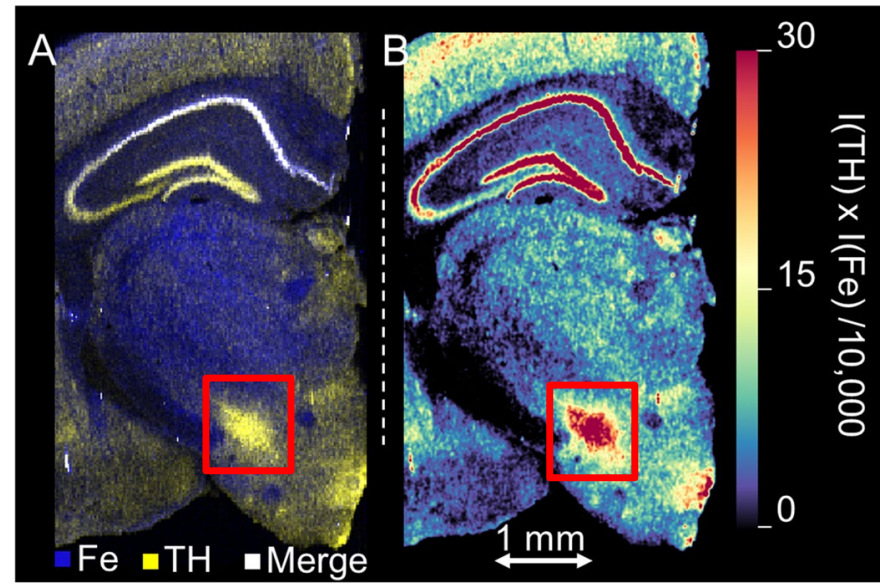

Figure 6. The co-localisation and correlation of Fe and TH was targeted with the high transmission method. (A) shows an overlay of Fe and TH and a high correlation is shown in white (CA subfields). (B) shows the Fe-TH score as a product of both intensities which can be used as an index in parkinsonian neurodegeneration. The red boxes indicate the area of the substantia nigra. mid-mass elements are transition metals which are known to have an important function in signal transmission and enzyme activity and may be used as biomarkers for neurological diseases.

Fig $6 \mathrm{~A}$ shows a high resolution ( $20 \mu \mathrm{m} \times 20 \mu \mathrm{m}$ per pixel) image of a murine brain containing parts of the limbic system and the substantia nigra. The iron distribution is shown in blue and $\mathrm{TH}$ in yellow. A high TH-Fe score (co-localisation) is shown in white and was particularly pronounced in the hippocampus where Fe mediates neuron development and memory function, and $\mathrm{TH}$ catalyses the formation of dopamine ${ }^{38}$.

TH and Fe have important functions in brain chemistry, but the co-localisation of dopamine and Fe can cause the production of reactive oxygen species. Previous reports have shown that the co-localisation of $\mathrm{TH}$ and $\mathrm{Fe}$ in the substantia nigra can be harnessed as an index for risk prediction of parkinsonian neurodegeneration ${ }^{27}$. The correlation of $\mathrm{TH}$ and Fe distribution may be visualised two ways: either, intensities can be overlayed as in Fig. 6A, or interlaced by multiplication, improving contrast of areas with both high Fe and high $\mathrm{TH}$ levels as shown in Fig. 6B. Improving the transmission of high-mass elements in iMSI while conserving SNRs for Fe produced enhanced contrasts and a higher dynamic range that was beneficial for visualisation of a TH-Fe index. As described elsewhere, the substantia nigra (shown in red box. Fig. 6(A) and (B)) has anatomical structures that have elemental and protein expressions that are highly concentrated in Fe and TH. The high transmission tune offers enhanced capabilities to target and investigate $\mathrm{Fe}$ and $\mathrm{TH}$ expression and co-localisation is these areas.

This method improved the analysis of endogenous elements and tyrosine hydroxylase as a proxy for the concentration of dopamine in murine brains in the region of the hippocampus and the substantia nigra. The method is applicable to any quadrupole-based ICP-MS related method and would also be of interest for analyses of higher mass elements operating close to 
the limits of detection, e.g. lanthanides in medicine and environmental samples $36,39,40$.

\section{Conclusions}

The complex structure, distribution of essential elements and expression of proteins in brain structures require specialised imaging approaches to provide high sensitivity while retaining highly resolved quantitative imaging. Standard tunes for iMSI via LA-ICP-MS offer a consistent performance over the entire mass range but can be adapted to provide higher transmission and therefore sensitivity for critical mass ranges by manipulation ion optics and quadrupole scanning parameters. This allows targeting of proteins that are of low abundance or express in micrometre scaled structures.

The adjustment of the scanning parameters of the quadrupole resulted in higher resolution for low masses, moderate change for endogenous mid mass elements, and lower mass resolution of high mass elements. The loss of mass resolution increased ion transmission improving SNRs by factor 6 for a ${ }^{172} \mathrm{Yb}$ labelled to anti-TH for IHC. This improvement was demonstrated by performing high-resolution imaging ( $25 \mu^{2}$ pixel resolution) of the murine hippocampal region. Unlike a standard tune, the high transmission mode provided high contrast images of the cornu ammonis and dentate gyros. The method was advantageous for visualisation of dopaminergic cells and the colocalisation of Fe allowing investigation of the TH-Fe index, a prediction tool used to estimate the risk for parkinsonian neurodegeneration.

High transmission methods are a valuable approach for quantitative imaging in $\mathrm{iMSI}$ and can also be transferred to other research areas where ICP-MS methods aim to determine heavy elements at their limits of analysis. Multiplexing approaches employing high transmission/low mass resolution methods are still possible if spectral overlap of mass peaks are avoided by choosing labels with several (6-8) mass units of difference.

\section{Conflicts of interest}

There are no conflicts to declare

\section{Acknowledgements}

D.C. is funded by the Deutsche Forschungsgemeinschaft (DFG. German Research Foundation) - 417283954. T.E.L. is supported by an Australian Government Research Training Program Stipend. P.A.D and P.A.A are recipients of an Australian Research Council Discovery Project (DP170100036).

We thank Lisa Bray for the preparation of mouse tissues. Images were analysed and created using imaging software by Robin Schmid (University of Muenster). The Florey Institute of Neuroscience and Mental Health acknowledges the strong support from the Victorian Government and in particular the funding from the Operational Infrastructure Support Grant.

\section{References}

1 L. Peris, M. Bisbal, J. Martinez-Hernandez, Y. Saoudi, J. Jonckheere, M. Rolland, M. Sebastien, J. Brocard, E. Denarier, C. Bosc, C. Guerin, S. Gory-Fauré, J. C. Deloulme, F. Lanté, I. Arnal, A. Buisson, Y. Goldberg, L. Blanchoin, C. Delphin and A. Andrieux, Nat. Commun., 2018, 9, 3775. P. Chaurand, M. E. Sanders, R. A. Jensen and R. M. Caprioli, Am. J. Pathol., 2004, 165, 1057-1068.

R. González de Vega, D. Clases, M. L. Fernández-Sánchez, N. Eiró, L. O. González, F. J. Vizoso, P. A. Doble and A. SanzMedel, Anal. Bioanal. Chem., 2019, 411, 639-646.

K. A. Giuliano and D. L. Taylor, Trends Biotechnol., 1998, 16, 135-140.

J. C. Lindon, E. Holmes and J. K. Nicholson, Expert Rev. Mol. Diagn., 2004, 4, 189-199.

6 R. Frank and R. Hargreaves, Nat. Rev. Drug Discov., 2003, 2 566.

7 B. A. Eijkelkamp, J. R. Morey, S. L. Neville, A. Tan, V. G. Pederick, N. Cole, P. P. Singh, C.-L. Y. Ong, R. Gonzalez de Vega, D. Clases, B. A. Cunningham, C. E. Hughes, I. Comerford, E. B. Brazel, J. J. Whittall, C. D. Plumptre, S. R. McColl, J. C. Paton, A. G. McEwan, P. A. Doble and C. A. McDevitt, PLOS Pathog., 2019, 15, e1007957. A. H. Coons, H. J. Creech and R. N. Jones, Proc. Soc. Exp. Biol. Med., 1941, 47, 200-202.

9 S.-R. Shi, Y. Shi and C. R. Taylor, J. Histochem. Cytochem., 2011, 59, 13-32.

10 J. A. Ramos-Vara, Vet. Pathol., 2005, 42, 405-426.

11 M. Nadji, M. Nassiri, V. Vincek, R. Kanhoush and A. R. Morales, Appl. Immunohistochem. Mol. Morphol. AIMM, 2005, 13, 277-282.

12 A. R. Dixon, C. Bathany, M. Tsuei, J. White, K. F. Barald and S. Takayama, Expert Rev. Mol. Diagn., 2015, 15, 1171-86.

13 E. C. Stack, C. Wang, K. A. Roman and C. C. Hoyt, Methods, 2014, 70, 46-58.

D. P. Bishop, N. Cole, T. Zhang, P. A. Doble and D. J. Hare, Chem. Soc. Rev., 2018, 47, 3770-3787.

Y.-H. Cheng, T. S.-C. Tam, S.-L. Chau, S. K.-M. Lai, H.-W. Tang, C.-N. Lok, C.-W. Lam and K.-M. Ng, Chem. Commun., 2019, 55, 2761-2764.

J. Seuma, J. Bunch, A. Cox, C. McLeod, J. Bell and C. Murray, Proteomics, 2008, 8, 3775-3784.

D. Pozebon, G. L. Scheffler and V. L. Dressler, J. Anal. At. Spectrom., 2017, 32, 890-919.

J. Qiao, K. Shi, X. Hou, S. Nielsen and P. Roos, Environ. Sci. Technol., 2014, 48, 3935-3942. D. Clases, R. Gonzalez de Vega, D. Bishop and P. Doble, Anal. Bioanal. Chem., 2019, 411, 3553-3560.

20 C. Zhang, F. Wu, Y. Zhang, X. Wang and X. Zhang, J. Anal. At. Spectrom., 2001, 16, 1393-1396.

21 L. Waentig, N. Jakubowski, S. Hardt, C. Scheler, P. H. Roos and M. W. Linscheid, J. Anal. At. Spectrom., 2012, 27, 1311. C. Giesen, H. A. Wang, D. Schapiro, N. Zivanovic, A. Jacobs, B. Hattendorf, P. J. Schüffler, D. Grolimund, J. M. Buhmann, S. Brandt, Z. Varga, P. J. Wild, D. Günther and B. Bodenmiller, Nat. Methods, 2014, 11, 417-422. 
D. Clases, R. Gonzalez de Vega, P. A. Adlard and P. A. Doble, J. Anal. At. Spectrom., 2019, 34, 407-412.

24 M. T. Westerhausen, T. E. Lockwood, R. Gonzalez de Vega, A. Röhnelt, D. P. Bishop, N. Cole, P. A. Doble and D. Clases, Analyst, 2019, 144, 6881-6888.

25 J. Lear, D. Hare, P. Adlard, D. Finkelstein and P. Doble, J. Anal. At. Spectrom., 2012, 27, 159. D. J. Hare, E. J. New, M. D. de Jonge and G. McColl, Chem. Soc. Rev., 2015, 44, 5941-5958. I. Volitakis, C. L. Masters, P. A. Adlard, R. A. Cherny, A. I. Bush, D. I. Finkelstein and P. A. Doble, Chem. Sci., 2014, 5, 2160-2169.

D. Berg, M. Gerlach, M. B. H. Youdim, K. L. Double, L. Zecca, P. Riederer and G. Becker, J. Neurochem., 2001. L. Zecca, M. B. H. Youdim, P. Riederer, J. R. Connor and R. R. Crichton, Nat. Rev. Neurosci., 2004, 5, 863-873. A. Hald and J. Lotharius, Exp. Neurol., 2005, 193, 279-290. W. Linert, E. Herlinger, R. F. Jameson, E. Kienzl, K. Jellinger and M. B. H. Youdim, Biochim. Biophys. Acta - Mol. Basis Dis., 1996, 1316, 160-168. S. Rao, L. Lago, R. de Vega, L. Bray, D. J. Hare, D. Clases, P. Doble and P. A. Adlard, Metallomics, 2020. K. L. Linge and K. E. Jarvis, Geostand. Geoanalytical Res., 2009, 33, 445-467. Acta - Part B At. Spectrosc., 2002, 57, 1361-1452. D. P. Bishop, D. Clases, F. Fryer, E. Williams, S. Wilkins, D. J. Hare, N. Cole, U. Karst and P. A. Doble, J. Anal. At. Spectrom., 2016, 31, 197-202. D. Clases, M. Sperling and U. Karst, Trends Anal. Chem., 2018, 104, 135-147. W. Marui, E. Iseki, M. Kato and K. Kosaka, Neurosci. Lett., 2003, 340, 185-188. Andrews, T. Schallert, H. Gunshin, M. K. Georgieff and A. Petryk, J. Nutr., 2009, 139, 672-679. and U. Karst, J. Trace Elem. Med. Biol., 2019, 51, 212-218. M. Birka, C. A. Wehe, O. Hachmöller, M. Sperling and U. Karst, J. Chromatogr. A, 2016, 1440, 105-111. 